\title{
Nuovo sistema canonico di elementi ellittici.
}

\author{
(Di T. Levi-Givita, a Padova.)
}

\section{INTRODUZIONE.}

$\mathrm{I}_{1}$ metodo della variazione delle costanti arbitrarie, dovuto a LAGRANGF (*), trova notoriamente le sue più cospicue applicazioni in meccanica celeste. Per lo studio del moto perturbato - di un punto $P$ soggetto all'attrazione preponderante di un centro $O$ - appare quasi sempre vantaggioso, secondo la teoria svolta sistematicamente dallo stesso LAGRANGF $\left({ }^{* *}\right)$, di sostituire ai sei elementi cartesiani, determinativi dello stato di moto di $P$ riferito ad $O$ (coordinate $x, y, z$ della posizione, componenti $p_{r}, p_{y}, p_{z}$ della quantità di moto), altrettante loro combinazioni che sarebbero (una al più eccettuata) costanti nel moto non perturbato. $\mathrm{E}$ precisamente sei parametri dell'ipotetica orbita ellittica, modernamente chiamata intermediaria, che sarebbe descritta da. $P$, qualora, cessando ogni influenza perturbatrice, esso si muovesse, a partire dallo stato di moto $\left(\begin{array}{lll}p_{x} & p_{y} & p_{z} \\ x & y & z\end{array}\right)$, sotto la esclusiva attrazione del corpo centrale.

Questi parametri (elementi ellittici) devono naturalmente indiriduare in modo diretto ed espressivo la forma e le dimensioni dell'orbita ellittica intermediaria, la sua posizione (rispetto agli assi di riferimento), la legge di percorrenza; ma la scelta comporta ancora molta arbitrarietà, sicchè conviene lasciarsi guidare da criteri di semplicità e di convenienza. I sei elementi tra-

$\left.{ }^{*}\right)$ Recherches sur les șuites récurventes dont les termes varient de plusieurs manières différentes, ou sur l'intégration des équations linéaires aux différences finies et partielles..., Oeuvres, T. IV, pp. 151-254. Cfr. le pagine 159-163.

(**) Théorie des variations séculaires des eléments des planètes e Théorie des variations périodiques ..., ibidem, 'I''. V, pp. 125-344, 345-489; Mémoire sur la thénie des variations des éléments des planètes, T. VI, pp. 713-768. 
dizionali degli astronomi sono: $a$ (semiasse maggiore), $e$ (eccentricità), $i$ (inclinazione), $\theta$ (longitudine del nodo ascendente), $\theta+g$ (longitudine del perielio), $l$ (anomalia media).

Nelle ricerche teoriche interessa che le formule di passaggio dalle $\left(\begin{array}{lll}p_{x} & p_{y} & p_{z} \\ x & y & z\end{array}\right)$ alle nuove variabili rientrino nel tipo canonico, donde l'introduzione, effettuata per la prima volta da $\mathrm{J}_{\mathrm{A}} \mathrm{coBr}$, di sestuple canoniche. Fissiamo l'attenzione sulla seguente, dovuta a Delaunay (*),

$$
\left.\begin{array}{lll}
L=\beta \sqrt{a}, \quad G=\beta \sqrt{a\left(1-e^{2}\right)}, \quad \Theta=\beta \sqrt{a\left(1-e^{2}\right)} \cos i & \begin{array}{l}
(\beta \text { costante che } \\
\text { dipende dalle } \\
\text { masse di } P \text { e } \\
\text { di } O)
\end{array} \\
l, \quad \theta, &
\end{array}\right\}
$$

da cui, con passaggi elementari, se ne desumono altre, per certi rispetti preferibili, segnalate da Pornciaré, e divenute ormai d'uso corrente col nome complessivo di variabili kepleriane $\left.{ }^{* *}\right)$.

In tutti questi sistemi, il parametro che fissa la posizione sull'orbita è: o proprio l'anomalia media $l$ [come in (l)], ovvero $l$ aumentata di angoli che nel moto ellittico sono costanti. Ne risulta una discreta complicazione nell'espressione della funzione perturbatrice; questa sarebbe noterolmente piì semplice se, al posto dell'anomalia media $l$, si usasse l'anomalia eccentrica $u$. Si può auzi dire che gli sviluppi in serie trigonometrica di $l$ si discutono teoricamente e si formano praticameute trasformando (con procedimenti più

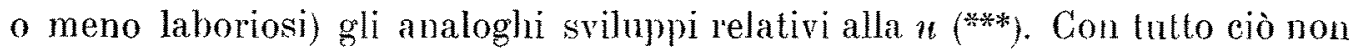
si adotta in definitiva la $u$, ma ci si attiene alla $l$, perchè da un lato questo argomento è il più indicato in quasi tutti i calcoli di prima approssimazione (rispetto alle masse perturbatrici); e d'alliro lato consente di conservare la forma canonica, il che giova sotto il duplice aspetto teorico e pratico, e non sarebbe raggiungibile (senza inconvenienti maggiori), effettuando un puro cambiamento di variabili sulla sestupla $\left(\begin{array}{ccc}L & G & \theta \\ l & g & \theta\end{array}\right)$.

(*) Cfr. L. Charlier, Die Mechanik des Himmels, B. I, p. 252.

(**) H. Poincari, Lecons de mécanique céleste, T. 1, pp. $4-13$; ovvero Charlinr, loco citato, pp. 292-294.

(***) Veggasi per es. il T. 2 (pp. 4-13 e Cap. XV) delle cilate Leçons... di Polncarí;

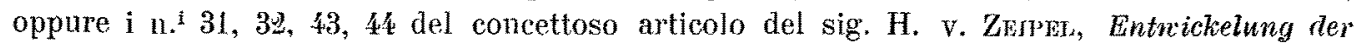
Stomungfunktion [Enc. der Math. Wiss., VI, 2, 13] 
Ma se si premette una lieve modificazione nella definizione dell'orbita ellittica intermediaria, si è senz'altro condotti - come apparirà dal presente scritto - ad un nuovo sistema canonico di elementi ellittici, che è sostanzialmente del tipo (I), salvo la sostituzione della anomalia eccentrica $u$ in luogo dell'anomalia media $l$.

La modificazione in parola non è più profonda (anzi ha proprio lo stesso ordine di grandezza) di quelle rispettivamente introdotte da JAcoBI e da Poincaré nel problema dei tre corpi. Come si sa, Jaсов ha sostituito, per uno dei tre corpi, alla attrazione del corpo centrale 0 , quella di un fittizio baricentro (di $O$ e del terzo corpo), inoltre alle masse reali masse fittizie (poco diverse); Poincarí ha invece mantenuto il vero corpo centrale, ma ha sostituito, per uno qualunque degli altri due, la quantità di moto relativa colla quantità di moto assoluta, modificando anch'egli le masse.

La nostra alterazione porterà esclusivamente sul coefficiente d'attrazione, cioè, possiamo dire, sulla massa del corpo centrale. Ecco in qual modo.

Consideriamo, per fissar le idee, il moto relativo di un punto $P$ rispetto ad $O$, nell'ipotesi che $P$ sia soggetto all'attrazione di $O$ e a forze perturbatrici qualisivogliono derivanti da un potenziale (funzione delle forze cambiata di segno) $\mu . F_{1}: \mu$ è un parametro numerico che serve a fissare l'ordine di grandezza. Sia $r$ la distanza $\overline{O P}, m$ la massa di $P, k_{0}$ l'attrazione newtoniana di $O$ su $P$, all'unità di distanza. Le equazioni del moto di $P$ sono canoniche rispetto alla sestupla $\left(\begin{array}{lll}p_{x} & p_{y} & p_{z} \\ x & y & z\end{array}\right)$ e ammettono per funzione caratteristica

$$
F=T-\frac{k_{0}}{r}+y \cdot F_{1}
$$

$T$ rappresentando la forza viva del mobile, ossia $\frac{1}{2 m}\left(p_{x}^{2}+p_{y}^{2}+p_{z}^{2}\right)$.

Secondo la definizione abituale, l'orbita intermediaria relativa ad un generico istante $t$ - nel caso attuale senz'altro osculatrice - è quella che sarebbe descritta da $P$, sotto l'attrazione $\frac{k_{0}}{r^{2}}$ di $O$, a partire dallo stato di moto $\left(\begin{array}{lll}p_{x} & p_{y} & p_{z} \\ x & y & z\end{array}\right)$ realmente posseduto da $P$ nel detto istante. Ciò presuppone naturalmente che si tratti di moto abbastanza poco diverso dall'ellittico perchè in particolare la differenza $T-\frac{k_{0}}{r}=h$ (energia del moto non perturbato) sia sempre negativa. Al variare di $t$, raria alquanto l'orbita osculatrice, e con essa la relativa energia $h$. 
Noi procederemo invece come segue. Sia $h_{0}$ il valore di $T-\frac{k_{0}}{r}$ nell'istante iniziale $t=t_{0}$. Per un altro istante qualsiasi $t$, definiamo $k$ mediante la posizione

$$
T-\frac{k}{r}=h_{0} \text {. }
$$

Una tale $k$ risulterà variabile coll'istante considerato, ma la sua differenza da $k_{0}$ sarà dell'ordine di $u$. (Per $u=0$ si ha infatti $h=h_{0}$, e quindi $k=k_{0}$, qualunque sia $t$ ).

Ciò premesso, nulla vieta di assumere come ellisse osculatrice, in un ge-

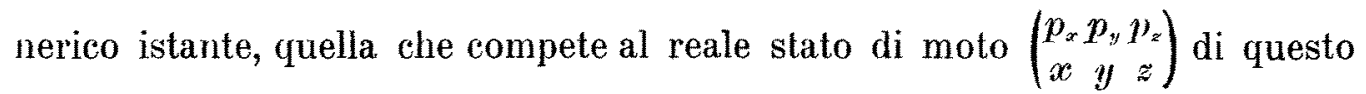
istante, nell'ipotesi però che la costante di attrazione del corpo centrale sia $k$, anzichè $k_{0}$.

Come si vede, mentre di solito (quando si mantiene alla costante di attrazione del centro $O$ il suo valore reale $k_{0}$ ) ci si lascia guidare da un' immagine che conserva esattamente la forza centrale (ma non l'energia $h$ del movimento ellittico tangente), colla nostra definizione si attribuisce bensì al coefficiente $k$ dell'attrazione newtoniana di $O$ un valore che varia alquanto dall'una all'altra delle ellissi osculatrici, ma viceversa si tiene costante per tutte il valore di $h_{0}$ : c' è quindi conservazione dell'energia (spettante ai vari moti tangenti). Per ciò queste orbite osculatrici e i relativi elementi ellittici possono opportunamente qualificarsi isoenergetici, a differenza degli ordinari che sono isodinamici.

Circa l'espressione trasformata che assumerà la funzione caratteristica $F$, quando vi si introducano i nuovi elementi, osserveremo che, seguendo identicamente dalla (III)

la (II) porge

$$
T-\frac{k_{0}}{r}=h_{0}+\frac{k-k_{0}}{r}
$$

$$
F^{r}=h_{0}+\frac{k-k_{0}}{r}+F_{1}
$$

Le cose vanno dunque come se alla funzione perturbatrice $\mu F_{1}$ si aggiungesse un termine complementare (che corrisponde a forza emanante dal centro) $\frac{k-k_{4}}{r}$ : la $k$ dipende (come si rileverà qui appresso) esclusivamente dall'asse maggiore dell'orbita osculatrice. 
$\$ 1$.

\section{Richiami concernenti il moto non perturbato}

\section{e la relativa equazione di Jacobi.}

Le equazioni del moto di un punto $P$, attratto dall'origine $O$ in ragione inversa del quadrato della distanza, sono, con manifesto significato dei simboli,

$$
\begin{aligned}
& m \ddot{x}+\frac{k x}{r^{3}}=0 \\
& m \ddot{y}+\frac{k y}{r^{3}}=0 \\
& m \ddot{z}+\frac{k z}{r^{3}}=0
\end{aligned}
$$

Introdotte le componenti

$$
p_{x}=m \dot{x}, \quad p_{y}=m \dot{y}, \quad p_{z}=m \dot{z}
$$

della quantità di moto di $P$, e la sua forza viva

$$
T=\frac{1}{2 m}\left(\mu_{x}^{2}+\mu_{y}^{*}+\nu_{z}^{2}\right)
$$

e posto

$$
H=T-\frac{k}{r}
$$

le (1), (2) equivalgono notoriamente al sistema canonico

$$
\left.\begin{array}{cc}
\dot{y}_{x}=-\frac{\partial H}{\partial x}, & \dot{p}_{y}=-\frac{\partial H}{\partial y}, \quad \dot{p}_{z}=-\frac{\partial H}{\partial z}, \\
\dot{x}=\frac{\partial H}{\partial p_{r}}, & \dot{y}=\frac{\partial H}{\partial p_{y}}, \quad \dot{z}=\frac{\partial H}{\partial p_{z}},
\end{array}\right\}
$$

avendosi in ogni caso l'integrale delle forze vive

$$
H=h \text {. }
$$

Riterremo negativa la costante $h$, con che classicamente si tratta di traiettorie ellittiche e moto kepleriano. 
Se nella (6) si risguardano le $p$ come simboli delle derivate di un'incognita funzione $W(x, y, z)$ a mezzo delle formule

$$
p_{r}=\frac{\partial W}{\partial x}, \quad p_{y}=\frac{\partial W}{\partial y}, \quad p_{x}=\frac{\partial W}{\partial z},
$$

si ha la corrispondente equazione di JACOBI. Ciascun suo integrale completo dà luogo ad una rappresentazione dell'integrale generale del sistema (5), ossia (nel caso $h<0$, cui esclusivamente ci riferiamo) degli $\infty^{6}$ moti ellittici, dovuti all'attrazione di 0 .

Prendiamo per es. l'integrale completo (con due costanti $G$ e $\theta$, oltre alla $h$ ) considerato da Porncaré nelle sue Leçons de mécanique céleste ${ }^{*}$ ).

Esso è

$$
W=\int_{i_{0}}^{r} R d r+G \zeta
$$

col seguente significato di $r_{0}, R$, e $\zeta$ :

$10^{\circ} r_{3}$ vi rappresenta la minima distanza di $P$ da $O$, ossia (dacchè si tratta di orbite ellittiche col fuoco in 0$) a(1-e)$, essendo $a$ il semiasse maggiore ed $e$ l'eccentricità.

2.

$$
R^{2}=m\left(2 h+\frac{2 k}{r}-\frac{G^{2}}{r^{2}}\right)
$$

con che $R$ contiene il solo argomento variabile $r$, dipendendo inoltre da $h$ e $k$, costanti intrinseche della (6), e dalla costante di integrazione $G$. Le due radici della equazione $r^{2} R^{2}=0$ (di secondo grado in $r$ ) corrispondono alla massima e minima distanza $a(1+e), a(1-e)$ del mobile dall'origine, donde le relazioni (fra radici e coefficienti)

$$
\left.\begin{array}{l}
2 a=-\begin{array}{l}
k \\
h
\end{array}, \\
a^{2}\left(1-e^{2}\right)=-\begin{array}{c}
a^{2} \\
2 h
\end{array}
\end{array}\right\}
$$

$3 .^{\circ} \%$ designa l'angolo del raggio vettore $O P$ colla linea dei nodi (intersezione, debitamente precisata quanto al verso, del piano dell'orbita col

(*) T. 1, pp. 66-69. 
piano di riferimento $O x y$ ). Come costanti di integrazione nell'espressione (8) di $W$ si risguardano la già menzionata $G$ e la longitudine $A$ ( $a$ priori qualunque) della linea dei nodi, che compare pel tramite di $\zeta$. Si ha infatti, essendo $\cos \theta, \sin \theta, 0$ i coseni direttori di tale linea, $\mathrm{e} \frac{x}{r}, \frac{y}{r}, \frac{z}{r}$ quelli di $O P$,

$$
\cos \zeta=\frac{x \cos \theta+y \sin \theta}{r} .
$$

Del resto non ci interessa la forma esplicita della funzione $\zeta(x, y, z, \theta)$ : basterà tener presente che essa dà luogo alla relazione

$$
\frac{\partial \stackrel{\varphi}{\partial \theta}}{\partial \theta}=-\cos i
$$

$i$ designando l'inclinazione (del piano dell'ellisse sul piano $O x y$ ).

Le equazioni in termini finiti delle traiettorie si ottengono ponendo

$$
\frac{\partial W}{\partial G}=g, \quad \frac{\partial W}{\partial \theta}=-\Theta
$$

con $g$ e $\Theta$ nuove costanti arbitrarie.

Il Loro significato risulta: per la seconda, dalla (-11), che dì

e cuindi

$$
\frac{\partial W}{\partial \theta}=\frac{\partial \%}{\partial \theta}=-\cos i
$$

$$
\Theta=G \cos i
$$

per la prima, riferendosi alla posizione perielia $r=r_{0}$, con che $R=0$ e

$$
\frac{\partial W}{\partial G}=\frac{\partial}{\partial G} \int_{r_{0}}^{r} R d r+\%
$$

si riduce a $\zeta: g$ rappresenta pertanto il valore di $\zeta$ quando il mobile transita pel perielio, ossia è l'angolo nodo-perielio.

Tutto ciò è puro riassunto di indispensabili premesse. Solo ora comincierò a staccarmi dal procedimento consueto, nell'intento di associare alle (12) un'equazione diversa dalla

$$
\frac{\partial W}{\partial h}=t-t_{n}
$$

che, da $J_{A C O B T}$ in poi, si fa sempre intervenire per completare la definizione del moto. 


\section{$\S 2$.}

La derivata di $W$ rispetto al parametro $l$.

Introduzione di un nuovo parametro $U$.

Dalle (8) e (9) segue ovriamente

$$
\frac{\partial W}{\partial k}=m \int_{r_{n}}^{r} \frac{d r}{r R} .
$$

L'integrale del secondo membro si calcola nel miglior modo sostituendo alla variabile di integrazione $r$ (che non è monotona, ove si segua il moto ellittico del punto $P$, ma oscilla continuamente fra la distanza perielia e l'afelia) la anomalia eccentrica $u$, che è sempre crescente e legata ad $r$ dalla nota relazione

$$
r=a(1-e \cos x)
$$

Si ha con ciò, portando nella espressione (9) li $R^{2}$ i valori di $k$ e di $G^{2}$ definiti ralle (10),

$$
r^{2} R^{2}=-2 h m\left\{-r^{2}+2 a r-a^{2}\left(1-e^{2}\right)\right\}=-2 h m a^{2} e^{2} \operatorname{sen}^{2} u
$$

dopo di che, ove si noti che $u$ è multiplo intero di $2 \pi$ per $r=r_{0}$ (e può quindi ritenersi nullo quando $r$ parte per la prima volta dal valore $r_{0}$ ), la (14) si riduce semplicemente a

$$
\frac{\partial W}{\partial k}=\sqrt{\frac{m}{-2 h}} n
$$

dovendosi attribuire al radicale il suo valore aritmetico.

Questa equazione, desunta per materiale derivazione dalla espressione (8) di $W$, sussiste identicamente in quanto, come abbiam fatto nel corso del calcolo, si tenga conto della (15). Qualora si conservi a $W$ la sua originaria accezione di funzione di $x, y, z$ (pel tramite di $r$ e di $\zeta$ ), e dei parametri $h, k, G, A$, la (14') diviene di necessitì equivalente alla (15) stessa; o meglio ne costi- 
tuisce la risolvente rispetto ad $u$, $a$ ed $e$ dovendosi intendere combinazioni di $h, k, G$ definite dalle (10).

$\grave{\mathrm{E}}$ opportuna una ulteriore trasformazione moltiplicativa, che sostituisca al parametro $k$ il nuovo parametro

$$
U=k \underbrace{m}_{-27} .
$$

Si può in conformitì risguardare $W$ come funzione dei sei argomenti

$$
x, y, z ; \quad U, G, \theta:
$$

a dir vero in $W$ interviene altresì il parametro $h$, ma non lo metto in evidenza, perchè lo ho trattato finora, e lo trafterò anche in seguito, come uma assoluta costante, a differenza di $G, \theta$ e $k$ - diciamo ormai $U$ - che mi riservo di considerare come variabili, che anzi ho già implicitamente falto variare, introducendo le derivate parziali di $W$. Con tale avertenza si ha dalle $\left(14^{\prime}\right)$ e (16)

$$
\partial W=u
$$

$\$ 3$.

\section{Risoluzione del sistema (14), (17) rapporto ad $x, y, z$.}

Il fatto che $W$ è integrale completo assicura notoriamente la risolubilità, rapporto ad $x, y, z$, del sistema costituito dalle due equazioni (14) delle traiettorie e da una terza equazione, che (in quanto si abbia riguardo al moto ellittico) contenga effettivamente il tempo. Tale è per certo la (17), tostochè vi si riguardi l'anomalia eccentrica $u$ come quella tal funzione di $t$, che caratterizza il moto ellittico a norma della equazione di KePI.ER. Così rimane provato che le $(14),(17)$ sono atte a definire $x, y, z$ in funzione, possiamo dire, della sestupla

$$
\left(\begin{array}{lll}
U & G & \Theta \\
u & g & \theta
\end{array}\right)
$$

Quanto alle formule esplicite, sarebbe inutile ricavarle più o meno laborio- 
samente dalle (14), (17): esse devono di necessità coincidere colle espressioni integrali spettanti alle $x, y, z$ nel moto ellittico, in funzione dei suddetti sei argomenti. Basta dunque nelle formule classiche, in cui intervengono abitualmente $a, e, i, g, \theta, u$, ritenere $a, e$ ed $i$ sostituiti mediante $U, G, \Theta$ a norma delle $(10),(16)$ e (13), che possono complessivamente scriversi (eliminando $k$ )

$$
\left.\begin{array}{l}
U=\sqrt{-2 h m} a, \\
G=\sqrt{-2 h m} a \sqrt{1-e^{2}}=U \sqrt{1-e^{2}}, \\
\Theta=\sqrt{-2 h m} a \sqrt{1-e^{2}} \cos i=G \cos i .
\end{array}\right\}
$$

Come si vede, $U$ è proporzionale all'asse maggiore, $G$ all'asse minore, il fattore di proporzionalità $\sqrt{-2 h m}$ dovendo trattarsi - ripetiamolo - come una costante assoluta.

Introducendo, per comodo di scrittura, le coordinate ausiliarie del mobile $*, p$ e $X, Y$ relative ad assi (sempre di origine $O$ ) situati nel piano dell'orbita e rispettivamente orientati : $1 .^{\circ}$ secondo la linea dei nodi e sua perpendicolare; ${ }^{\circ}{ }^{\circ}$ secondo gli assi dell'ellisse, si hanno le note formule elementari :

$$
\left.\begin{array}{l}
x=\cos \theta \mathfrak{x}-\cos i \sin \theta \mathfrak{y}=\cos \theta \mathfrak{x}-\frac{\Theta}{G} \cdot \sin \theta \mathfrak{y}, \\
y=\sin \theta \mathfrak{X}+\cos i \cos \theta \mathfrak{y}=\sin \theta \mathfrak{x}+\frac{\Theta}{G} \cos \theta \mathfrak{y}, \\
z=\quad \sin i \mathfrak{y}=\sqrt{1-\frac{\Theta^{2}}{G^{2}}} \mathfrak{y} ; \\
\mathfrak{x}=\cos g X-\sin g Y, \quad \mathfrak{y}=\sin g X+\cos g Y ; \\
X=a(\cos u-e), \quad Y=a \sqrt{1-e^{2}} \sin u,
\end{array}\right\}
$$

le ultime delle quali, attese le (18), assumono l'aspetto

$$
X=\frac{1}{\sqrt{-2 h m}}\left|U \cos u-\sqrt{U^{2}-G^{2}}\right|, \quad Y=\frac{1}{\sqrt{-2 h m}} G \sin u .
$$

Ove si immagini di portare successivamente nelle (19) le (20) e (21), si ottengono le risolventi delle (14), (17) sotto la forma voluta

$$
\left.\begin{array}{l}
x=x(U, G, \Theta ; u, g, \theta ; h), \\
y=y(U, G, \Theta ; u, g, \theta ; h), \\
\approx=\approx(U, G, \Theta ; u, g, \theta ; h) .
\end{array}\right\}
$$


Se ne trae, per le componenti della quantità di moto (dei movimenti kepleriani definiti da queste equazioni),

$$
p_{x}=m \dot{x}=m \frac{\partial x}{\partial u} \dot{u}, \quad p_{y}=m \frac{\partial y}{\partial u} \dot{u}, \quad p_{z}=m \frac{\partial z}{\partial u} \dot{u}
$$

$\dot{u}$ dovendo desumersi dall'equazione di KEPLER

$$
u-e \sin u=n t+\text { cost. }
$$

in cui il moto medio $n$ vale $\sqrt{\frac{k}{m a^{3}}}$, ossia, per la prima delle $(10)$,

$$
\frac{1}{a} \sqrt{-\frac{2 h}{m}}
$$

Si ha cosi, badando anche alla (15),

$$
\dot{u}=\frac{n}{1-e \cos u}=\frac{1}{r} \sqrt{-\frac{2 h}{m}}
$$

e in conformità

$$
\left.\begin{array}{l}
p_{x}=\frac{\sqrt{-2 \hbar m}}{r} \frac{\partial x}{\partial u}, \\
p_{y}=\frac{\sqrt{-2 h m}}{r} \frac{\partial y}{\partial u} \\
p_{z}=\frac{\sqrt{-2 h m}}{r} \partial z \\
\partial u
\end{array}\right\}
$$

Queste costituiscono evidentemente anche le espressioni integrali di $p_{x}, p_{y}, p_{z}$, considerate come incognite ausiliarie delle equazioni del moto [sotto la forma canonica (5)], semprechè vi si risguardino: $h, G, \Theta, g, \theta$ come cinque costanti arbitrarie di integrazione (la sesta essendo inclusa in $u$ ), $k$ come fisso, ma $s u$ scettibile ai valore qualsiasi, anzi sostituito da $U=k \sqrt{-\frac{m}{-2 h}}$ a norma della (16). Ora le stesse espressioni integrali di $p_{x}, p_{y}, p_{z}$ devono trarsi dalle (7) (attesa la (ircostanza che $W$ è integrale completo), in quanto vi si intendano per $x, y, z$ le relative espressioni integrali (22) (colla accezione ora detta delle lettere $h, G, \Theta, g, \theta, u, L)$. Le (7) equivalgono pertanto alle (23) (previa la sostituzione delle $x, y, z)$, qualunque siano i valori dei sette argomenti $U, G, \Theta, u, g, \theta, h$. 
Badando alla già rilevata circostanza che le (22) non sono altro che le risolventi delle (14), (17), si conclude che le (22), (23) equivalgono complessivamente alle (14), (17), (7).

\section{$\$ 4$.}

\section{Constatazione della canonicità.}

Le (22), (2:3) definiscono sotto forma esplicita una trasformazione della sestupla.

$$
\left(\begin{array}{lll}
p_{x} & p_{y} & p_{z} \\
x & y & z
\end{array}\right)
$$

nella

$$
\left(\begin{array}{lll}
U & G & \Theta \\
u & g & \theta
\end{array}\right)
$$

dipendente da un parametro $h$. Dico che (qualunque sia il valore attribuito ad $h$ ) la trasformazione è canonica, le nuove variabili coniugate essendo $U, u ; G, g ; \Theta, \theta$.

All'uopo basta sfruttare la circostanza che le (14), (17), (7), e con esse la relazione differenziale

$$
\begin{gathered}
d W=\frac{\partial W}{\partial x} d x+\frac{\partial W}{\partial y} d y+\frac{\partial W}{\partial z} d z+\frac{\partial W}{\partial U} d U+\frac{\partial W}{\partial G} d G+\frac{\partial W}{\partial \theta} d \theta= \\
p_{x} d x+p_{y} d y+p_{z} d z+u d U+g d G-\Theta d \theta
\end{gathered}
$$

sono necessaria conseguenza delle formule di trasformazione (22), (23). Ne consegue ulteriormente, togliendo da una parte e dall'altra $d(U u+G g)$,

$$
d(W-U u-G g)=\left(p_{x} d x+p_{y} d y+p_{z} d z\right)-(U d u+G d g+\Theta d \theta) .
$$

La differenza dei due trinomi

$$
p_{x} d x+p_{y} d y+p_{z} d z \quad \text { e } \quad U d u+G d g+\Theta d \theta
$$

è dunque un differenziale esatto, c. d. $d$. 
$\S \check{5}$

Confronto fra i nuovi elementi e quelli di Delaunay --- Conseguente espressione del momento delle quantità di moto - Identità di comportamento di fronte alle trasformazioni di Poincaré.

La trasformazione canonica abitualmente adottata fa passare dalla sestupla cartesiana alla

$$
\left.\begin{array}{lll}
L=\sqrt{k} \bar{m} \sqrt{a} & \theta=I \sqrt{T-\overline{e^{2}}} & \Theta=G \cos i \\
l \text { (anomalia media) } & g & \theta,
\end{array}\right\}
$$

in cui $k$ (attrazione di $O$ all'unità di distanza) va trattata come un parametro costante. Per questo fatto, la sestupla di Delaunay può dirsi isodinamica (come già si accennò nell'Introduzione), e contrassegnarsi con (D). In modo analogo sarà a dirsi isoenergetica, e si indicherà con (E) la nostra sestupla.

$$
\left.\begin{array}{lll}
U=\sqrt{-2} h \vec{m} a & \theta=U \sqrt{1-e^{2}} & \Theta=G \cos i \\
u \text { (anomalia eccentrica) } & g & \theta,
\end{array}\right\}
$$

nella quale si tratta come costante l'energia $h$.

Gli elementi $g$ e $\theta$ hanno identico significato in (D) ed in (E). Il divario essenziale consiste nell'intervento dell'anomalia media in (D), e della eccentrica in (E). Ma vi sono altre differenze secondarie. La variabile coniugata all'anomalia dipende bensi, in entrambi $\mathrm{j}$ casi, dal solo asse maggiore dell'orbita osculatrice: però, mentre $L \grave{e}$ proporsionale $a \sqrt{a}, U$ è addirittura proporzionale ad a. La coniugata di $g$, designata in ambedue le sestuple con $G$, $\grave{e}$ proporzionale al parametro dell'orbita in (D), al semiasse minore in $(\mathrm{E})$.

Importa notare che, se si tratta di una stessa orbita, la prima delle (10) dà fra le due quantità $h$ e $k$ la relazione

talchè

$$
2 a=-\frac{k}{h},
$$

$$
\sqrt{k m a}=\sqrt{-2 \hbar m} a \text {. }
$$


Coincidono quindi [in base alle tabelle (D) ed (E)] i valori numerici di $L$ e di $U$; per conseguenza anche $G$ ha un medesimo valore nei due casi, e così $\Theta$. Ne discende ulteriormente che $G$ conserva la sua abituale interpretazione di momento risultante delle quantità di moto; seguitano perciò a sussistere, anche per gli elementi $(\mathrm{E})$, le note formule

$$
\left.\begin{array}{l}
y p_{z}-z p_{y}=G \sin i \sin \theta \\
z p_{x}-x p_{z}=-G \sin i \cos \theta \\
x p_{y}-y p_{x}=\quad G \cos i
\end{array}\right\}
$$

in cui $i$ si deve intendere eliminato a mezzo della $\Theta=G \cos i$.

E appena necessario aggiungere che, applicando alla sestupla (E) le trasformazioni canoniche indicate da PoIncaré per la (D), si raggiunge analogamente l'intento di introdurre variabili appropriate alle piccole eccentricità ed inclinazioni. Si hanno così successivamente le sestuple

$$
\left.\begin{array}{ccc}
U & p_{1}=U-G & p_{2}=G-\Theta, \\
\omega=u+g+\theta & \omega_{1}=-(g+\theta) & \omega_{2}=-\theta,
\end{array}\right\}
$$

con evidente significato degli angoli $\omega, \omega_{1}, \omega_{2}$.

$\$ 6$.

Introduzione dei nuovi elementi nel problema dei tre corpi.

Sịeno $O, P, P^{\prime}$ i tre corpi ( $O$ corpo centrale di inassa preponderante); $m_{0}, m, m^{\prime}$ le rispettive masse; $x, y, z ; x^{\prime}, y^{\prime}, z^{\prime}$ le coordinate di $P$ e di $P^{\prime}$, riferite ad assi di orientazione fissa coll'origine in $O ; f$ la costante di attrazione universale; $r=\overline{O P}, r^{\prime}=\overline{O P^{\prime}}, \Delta=\overline{P P^{\prime}}$ le tre distanze; $p_{x}, p_{y}, p_{z}$ le componenti della quantità di moto assoluta di $P ; p_{r}^{\prime}, p^{\prime}, p_{z}^{\prime}$ le componenti della analoga quantità di moto di $P^{\prime}$. 
Posto

$$
\begin{aligned}
H_{0} & =\frac{1}{2}\left(\frac{1}{m}+\frac{1}{m_{0}}\right)\left(p_{x}^{2}+p_{y}^{2}+p_{z}^{2}\right)-f \frac{m_{0} m}{r} \\
H^{\prime} & =\frac{1}{2}\left(\frac{1}{m}+\frac{1}{m_{0}}\right)\left(p_{x}^{\prime 2}+p_{y}^{\prime z}+p_{z}^{\prime 2}\right)-f \frac{m_{0} m^{\prime}}{r^{\prime}} \\
F_{0} & =H_{0}+H_{0}^{\prime}, \\
\mu_{0} F_{1} & =\frac{1}{m_{0}}\left(p_{x} p_{x}^{\prime}+p_{y} p_{y}^{\prime}+p_{z} p_{z}^{\prime}\right) \cdots f \frac{m m^{\prime}}{\Delta},
\end{aligned}
$$

le equazioni del moto relativo, sotto la forma canonica di Porncaré, ammettono per funzione caratteristica

$$
F=F_{0}+\mu_{\cdot} F_{\cdot}^{\prime},
$$

essendo variabili coniugate le coordinate di ciascuno dei due corpi $P$ e $P^{\prime}$ e le corrispondenti componenti delle loro quantita di moto; $\mu F_{1}$ costituisce la funzione perturbatrice, e più precisamente il termine $-f \frac{m m^{\prime}}{\Delta}$ ne è la parte principale, il trinomio $\frac{1}{m m_{0}}\left(p_{x} p_{x}^{\prime}+p_{y} p_{y}^{\prime}+p_{z} p_{z}^{\prime}\right)$ il termine complementare.

A ciascuna delle due sestuple

$$
\begin{aligned}
& \left(\begin{array}{lll}
p_{x} & p_{y} & p_{z} \\
x & y & z
\end{array}\right), \\
& \left(\begin{array}{lll}
p^{\prime} & p^{\prime} & p_{z}^{\prime} \\
x^{\prime} & y^{\prime} & z^{\prime}
\end{array}\right)
\end{aligned}
$$

conviene sostituire degli elementi ellittici, definiti in base ad ipotetici moti non perturbati.

L'abituale criterio isodinamico porta a valersi di due sistemi canonici ausiliari aventi rispettivamente $H_{0}$ e $H_{0}^{\prime}$ per funzioni caratteristiche.

Noi faremo invece corrispondere ad ogni istante due funzioni poco diverse dalle $H_{0}, H_{0}^{\prime}$ relative allo stesso istante, cioè

$$
\left.\begin{array}{l}
H=\frac{1}{2}\left(\frac{1}{m}+\frac{1}{m_{0}}\right)\left(p_{x}^{2}+p_{y}^{2}+p_{z}^{2}\right)-\frac{k}{r}, \\
H^{\prime}=\frac{1}{2}\left(\frac{1}{m^{\prime}}+\frac{1}{m_{0}}\right)\left(p_{x}^{\prime 2}+p_{y}^{\prime 2}+p_{z}^{\prime 2}\right)-\frac{k^{\prime}}{r^{\prime}},
\end{array}\right\}
$$


modificando (lievemente) i coefficienti di $\frac{1}{r}, \frac{1}{r^{\prime}}$ in confronto dei valori $f m_{n} m$, $f m_{0} m^{\prime}$ che loro competono in $H_{0}, H_{0}^{\prime}$. Questi coefficienti $k$ e $k^{\prime}$ li intenderemo fissati in guisa che $H$ e $H^{\prime}$ si conservino, nel moto reale di $P, F^{\prime}$, costantemente eguali ai loro valori iniziali $h_{0}, h_{0}^{\prime}$.

Ciò posto, riportandoci ai precedenti paragrafi, potremo coordinare al sistema canonico di funzione caratteristica $H$ una trasformazione canonica, che fa passare dalla sestupla (29) ad una sestupla

$$
\left(\begin{array}{lll}
U & G & \ominus \\
u & g & \theta
\end{array}\right)
$$

di elementi ellittici $(E)$, corrispondenti all' orbita intermediaria che sarebbe descritta dal punto $P$, a partire dal suo reale stato di moto dell' istante $t$, qualora cessasse ogni perturbazione. In questo moto ipotetico [come risulta dal confronto dell'espressione (31) di $H$ colle (3), (4)]:

1. ${ }^{\circ}$ funge da massa di $P$

$\frac{1}{\frac{1}{m}+\frac{1}{m_{0}}}=\frac{m}{1+\frac{m}{m_{0}}}$ (poco diversa dalla massa reale $m$ per essere piccolo il rapporto $\left.\frac{m}{m_{0}}\right)$;

2.0 il vettore $\left(p_{x}, p_{y}, p_{*}\right)$, che concorre a definirlo (e che è, nel moto reale di $P$, la quantità di moto assoluta dell' istante cui si riferiscono gli elementi) si interpreta come quantità di moto relativa, talchè l'orbita intermediaria non riesce esattamente osculatrice;

$3 .^{\circ}$ (e qui risiede la differenza in confronto della definizione ordinaria) l'attrazione del centro $O$ ha un coefficiente $k$ (poco diverso da $f m_{0} m$ ), scelto in guisa che risulti $H=h_{0}$.

Analogamente per $P^{\prime}$.

Ove si badi alla (16) e si noti che [a norma delle (27) e (31)]

$$
H_{0}-H=\frac{k-f m_{n} m}{r},
$$

si vede che la sostituzione degli elementi ellittici isoenergetioi in $H_{0}$ Ia riduec a

$$
h_{0}+\frac{\sqrt{\frac{-2 h_{0}}{m} U-f m_{0} m}}{r}
$$


Del pari, designando gli elementi di $P^{\prime}$ con lettere accentate, $H_{0}$ si riduce a

$$
h_{0}^{\prime}+\frac{\sqrt{\frac{-2 h_{0}^{\prime}}{m^{\prime}}} U^{\prime}-f m_{0} m^{\prime}}{r} .
$$

Insomma, designando con $C, \alpha, \alpha^{\prime}, \beta, \beta^{\prime}$ costanti positive, che dipendono soltanto dalle masse e dalle condizioni iniziali, si ha

$$
F_{0}=-C+\frac{\alpha U-\beta}{r}+\frac{\alpha^{\prime} U^{\prime}-\xi^{\prime}}{r^{\prime}}
$$

coll'avvertenza che $r$ ed $r^{\prime}$ si devono ritenere espresse per mezzo di $U, G, u$, o rispettivamente $U^{\prime}, G^{\prime}, u^{\prime}$, a norma delle (15) e (18).

Resta la funzione perturbatrice $\mu F_{1}$. E appunto nei suoi riguardi che si fa palese il vantaggio dei nuovi elementi, i quali comprendono l'anomalia eccentrica $u$, al posto della media, e consentono così sia di attribuire a $\mu . F_{1}$ espressione esplicita (mentre di solito c'è di mezzo l'equazione di KEPLER), sia più agevoli sviluppi in serie. Ciò è troppo noto perchè occorra illustrarlo. Terminerò osservando che, anche coi nuovi elementi, dei due gruppi di formule di trasformazione destinati ad introdurli [(22) e (23)], viene in uso soltanto il primo per la parte principale $-f \frac{m m^{\prime}}{\Delta}$, soltanto il secondo per il termine complementare $\frac{1}{m_{0}}\left(p_{x} p_{x}^{\prime}+p_{y} p_{y}^{\prime}+p_{z} p_{z}^{\prime}\right)$. 